\title{
In-Band Full-Duplex Radar-Communication System
}

\author{
Seyed Ali Hassani ${ }^{\circledR}$, Student Member, IEEE, Vesa Lampu, Karthick Parashar, Lauri Anttila ${ }^{\circledR}$, Member, IEEE, \\ André Bourdoux, Member, IEEE, Barend van Liempd ${ }^{\circledR}$, Member, IEEE, Mikko Valkama ${ }^{\circledR}$, Senior Member, IEEE, \\ François Horlin ${ }^{\circledR}$, Member, IEEE, and Sofie Pollin ${ }^{\circledR}$, Senior Member, IEEE
}

\begin{abstract}
In-band full-duplex (IBFD) technology is a promising solution to boost the throughput of wireless networks. To bring IBFD to reality, the modem has to cancel the self-interference (SI) signal, which includes the strong direct Tx leakage signal and the weaker reflected Tx signal from the surroundings. Adaptive analog and digital SI cancelation schemes have been proposed. It becomes then interesting to understand, although, how the echoed SI could be exploited for enabling radar functionality while reusing the waveform and the already-existing hardware. This article formulates the monostatic radar system model starting from the communication system model. Beside simulation-based assessment, the performance is also evaluated by an IBFD system prototype, which consists of both analog and digital SI canceller modules, enabling $>85 \mathrm{~dB}$ Tx-Rx isolation. The system is enhanced with Doppler radar functionality, reusing as much as possible the existing IBFD functional blocks. The experimental result shows the accuracy of the proposed system to measure the velocity of mobile objects at various speeds between 0.2 and $1 \mathrm{~m} / \mathrm{s}$ while the device is simultaneously served as a node to perform in-band bidirectional communication. This ability suits the proposed system for a broad spectrum of opportunistic remote sensing applications, such as body and hand gesture detection.
\end{abstract}

Index Terms-Hand and body gesture detection, in-band full duplex (IBFD), monostatic Doppler radar, opportunistic remote sensing, self-interference (SI) cancelation, wireless sensing.

\section{INTRODUCTION}

$\mathbf{T}$ ODAY, the use of radios is continuously increasing in commercial and industrial electronic devices and has caused the congestion of the available radio spectrum. This motivates implementing multiple functionalities in such a way that they share the same frequency band and potentially the same hardware platform. A good example is opportunistic radio frequency (RF) sensing, which has been studied extensively to offer a

Manuscript received October 22, 2019; revised April 4, 2020; accepted April 29,2020 . This work was supported by the European Union's Horizon 2020 under Grant Agreement 732174 (ORCA project). (Corresponding author: Seyed Ali Hassani.)

Seyed Ali Hassani and Sofie Pollin are with the Electrical Engineering, KU Leuven, 3000 Leuven, Belgium (e-mail: seyedali.hassani@kuleuven.be; sofie.pollin@esat.kuleuven.be).

Vesa Lampu, Lauri Anttila, and Mikko Valkama are with the Electrical Engineering, Tampere University of Technology, 33720 Tampere, Finland (e-mail: vesa.lampu@tuni.fi; lauri.anttila@tuni.fi; mikko.valkama@tuni.fi).

Karthick Parashar was with the imec Leuven, 3001 Leuven, Belgium. He is now an independent researcher (e-mail: karthick.np@gmail.com).

André Bourdoux and Barend van Liempd are with the imec Leuven, 3001 Leuven, Belgium (e-mail: andre.bourdoux@imec.be; barend.vanLiempd@ imec.be).

François Horlin is with the Universit Libre de Bruxelles, 1050 Bruxelles, Belgium (e-mail: fhorlin@ulb.ac.be).

Digital Object Identifier 10.1109/JSYST.2020.2992689 promising alternative to traditional sensing solutions, such as video surveillance and wearable sensors, that do not respect users' privacy and comfort.

In opportunistic wireless sensing technology, the already existing electromagnetic waves are reused to extract the environmental context. Such an approach enables a wide range of applications, such as localization, e-healthcare, through-the-wall tracking, and body and hand gesture recognition. Via embedding the latter capability into smartphones, for instance, the users can go beyond touchscreens by interacting with the device through gestures in the space around it.

There is a substantial body of literature in which the received signal strength indicator (RSSI) of the ambient Wi-Fi signal has been adopted as a metric to perform indoor localization [1], [2] and passive human activity/gesture recognition [3]-[6]. Since the temporal and spatial variance can quickly distort the RSSI criteria [7], the recent attempts have focused on extracting the Doppler/velocity, mostly in the form of passive bistatic Doppler radar. In such a scheme, the radar correlates the received signal from the line of sight of the source of opportunity (reference signal) with the echo from the target (surveillance signal).

Multiple wireless devices are required to establish a passive bistatic Doppler radar system. The introduced sensing system in [8] enables human gesture recognition by employing multiple Wi-Fi transmitters and one surveillance receiver. Similar works are represented in [9]-[12], which also need a Wi-Fi access point (AP) and a dual-channel receiver. To improve radar performance, Tan and Woodbridge [13] made use of GPS-based clock synchronization to synchronize the reference and the surveillance radios. In [14], a directional coupler and two directional antennas are employed to reduce the requirements to one receiver and one Wi-Fi AP.

There are following two fundamental constraints in these prior art opportunistic remote sensing techniques.

1) The performance of bistatic passive radars is often sensitive to the isolation of the reference and surveillance channels. Beam antennas have been used to overcome this issue while it narrows the system angle of view (AOV). To extend the AOV, multiple directional surveillance receivers have to be employed [13]. This, consequently, increases the realization cost and implementation complexity.

2) The transmitted signal in the scenarios mentioned above has a noncontinuous nature. Thus, in addition to the clock synchronization between the reference and the surveillance receivers, a second mechanism is required to synchronize the transmitter of opportunity and the 


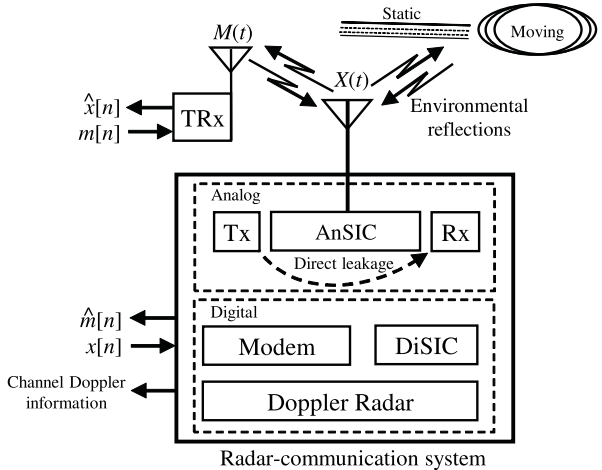

Fig. 1. Overview of the proposed radar-capable IBFD communication device. While the transmit signal $X(t)$ carries information to the other party, the IBFD device can also opportunistically make use of the environmental reflections of $X(t)$ to extract the Doppler state of the channel.

bistatic radar. For example, a synchronization technique is presented in [10] to deal with the noncontinuity of the Wi-Fi signal and to reduce the impact of the large beacon interval.

In [15], we addressed the issues mentioned above by introducing a radar-capable $\mathrm{Tx}-\mathrm{Rx}$ architecture, in which the already existing components of the in-band full-duplex (IBFD) communication platform are reused to perform wireless sensing simultaneously. This approach does not suffer from the sensitive process of over-the-air reference signal reconstruction and facilitates explicit synchronization between the radar illuminator and the surveillance receiver. We also introduced a proof of concept prototype in [16], in which an analog self-interference (SI) isolation circuitry is employed to allow monostatic Doppler detection via processing the environmental echoes of the self-transmit communication signal. Next, we outline the contributions of this article that extend our previous studies in [15] and [16] to further enhance the sensing performance in a real-world IBFD communication link.

\section{A. Contributions}

Fig. 1 shows the proposed radar-communication (RadCom) system operating in a full-duplex mode where it forms an inband bidirectional link with another communication terminal, shown by the TRx block. As shown, the system benefits from an analog SI cancelation (AnSIC) module that provides sufficient $\mathrm{Tx}-\mathrm{Rx}$ isolation, which is necessary before digital sampling. In addition, a digital SI cancelation (DiSIC) unit is embedded in the system. The key contributions in this article are summarized as follows.

1) First, we write the monostatic IBFD Doppler radar system model starting from the IBFD communication model. We expand the model in [15] and [16] by deriving a detailed mathematical model, including both analog and digital subsystems. The model determines the narrowband Doppler signal and identifies the different interference components. Via simulations, measurements, and analysis, we show that the self and environmental signal interference for a joint radar and communication system can be resolved.
2) Second, we implement an IBFD platform that achieves larger than $85-\mathrm{dB}$ SI rejection, jointly by analog and digital modules, and enables in-band bidirectional communication. Our design also utilizes the DiSIC block to improve the radar by further suppression of the direct SI signal. To this end, we enhance the prototype in [16] by a real-time DiSIC realization, showing that the static analog and hybrid digital filtering enables simultaneous communication and radar functionality for a range of communication distances and Doppler detection speeds.

3) Finally, we study the impact of the adaptive analog and digital SI cancelation. The experimental analysis shows that dynamic tuning of the SI cancellers can remove the Doppler information from the received SI. Hence, the ana$\log$ and digital SI cancelation coefficients should remain unchanged during the epoch over which the Doppler signal is computed. As this epoch is typically long compared to a channel coherence time, it is not efficient to fix the coefficients from the communication point of view. We, however, show that it is possible to keep the analog SI cancelation static, while having static and dynamic filters for the digital SI cancelation.

The remainder of this article is structured as follows. Section II briefly introduces the IBFD technology. In Section III, we develop the mathematical system model followed by distortion analysis focusing on the first contribution. The system is then simulated in Section IV giving insight in the joint analog and digital SI cancelation that is needed to get a sufficient signal-tonoise ratio (SNR) for the Doppler signal. For contribution 3, we focus on the AnSIC and DiSIC schemes that are implemented in our SDR prototype, as discussed in Section V. The experimental results are demonstrated in Section VI. Section VII discusses the system limitations and some practical aspects, and finally the conclusion is drawn.

\section{SI CHALLENGE IN IBFD COMMUNICATION}

The IBFD technology is well known for its potential to duplicate the link throughput as it allows transmission and reception over the same frequency band at the same time. In fact, this is not the only benefit with IBFD since it also allows the device to recapture the environmental reflections of what it transmits. From this point of view, an IBFD radio can be seen as an integrated radar illuminator and receiver.

There are two interfering replicas of the self-transmit signal: first, the analog direct-path leakage shown by the dashed arrow in Fig. 1; and second, the signal reflecting off device-extrinsic scatters, i.e., the static and moving objects in the vicinity.

The direct SI signal is orders of magnitude stronger than the desired low-power signal coming from a remote communication node, to exemplify, $104 \mathrm{~dB}$ in a typical Wi-Fi scenario [17]. This can dramatically reduce the reception sensitivity, or more likely saturates the receiver chain. To enable communication in an IBFD scheme, therefore, the interfering signal has to be suppressed nearly to the noise floor.

To overcome this challenge, typical IBFD architectures make use of an AnSIC block, which guarantees adequate Tx-Rx 


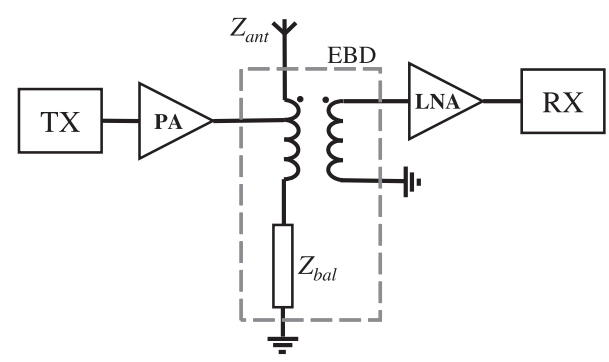

Fig. 2. Structure of a typical EBD-based AnSIC. High SI rejection can be achieved by the electrical balancing of the antenna impedance $Z_{\text {ant }}$ and the dummy load $Z_{\text {bal }}$.

isolation prior to analog-to-digital conversion (ADC). Once the baseband received signal is digitized, further SI suppression is possible in an additional stage by a DiSIC module.

A real-world IBFD realization has not been possible without the recent advances in SI cancelation technology. The represented active AnSIC techniques in [18] and [19], for instance, allow for up to $70-\mathrm{dB}$ SI cancelation at the RF stage. On the digital side, Korpi et al. [20], [21] suggested a scheme in which they establish a nonlinear adaptive design to model the impact of the multipath channel and the hardware imperfections. Their proposed approach estimates the residual SI after analog SI attenuation and enhances the sensitivity by subtracting a correction signal from the received baseband signal.

\section{A. Electrical Balance Duplexing}

An electrical balance duplexer (EBD) is a kind of AnSIC scheme that offers impressive linearity and insertion loss performance. In contrast with the nontunable counterparts, such as fixed-frequency surface-acoustic wave filters, this AnSIC technique allows us for scaling and frequency flexibility. Furthermore, using silicon-on-insulator technology, the EBD can be optimally deployed on-chip, which appropriates it for mobile wireless platforms. In the proposed design in this work, the EBD is the key enabling element as it can significantly suppress the direct Tx leakage to allow the reception of the environmental SI reflection, which is needed for the Doppler radar.

Fig. 2 depicts the topology of a single-ended EBD. This RF SI cancelation concept is based on hybrid transformers providing signal cancelation through the electrical balancing of two impedances: the antenna impedance $Z_{\text {ant }}$ and an on-chip dummy load called the balance network $Z_{\text {bal. }}$. Applying the ideal $S$-parameters of a symmetrical hybrid (i.e., when the transformer tapping ratio is $r=1$ ), the Tx-Rx isolation $I$ can be obtained as follows:

$$
I=20 \log _{10}\left|\Gamma_{\text {ant }}-\Gamma_{\text {bal }}\right|-6.02
$$

where $\Gamma_{\text {ant }}$ and $\Gamma_{\text {bal }}$ are, respectively, the complex reflection coefficients of the balancing impedance and the antenna impedance defined as

$$
\Gamma_{\text {ant }}=\frac{Z_{\text {ant }}-R_{o}}{Z_{\text {ant }}+R_{o}} \text { and } \Gamma_{\text {bal }}=\frac{Z_{\text {bal }}-R_{o}}{Z_{\text {bal }}+R_{o}}
$$

where $R_{o}$ is the output load.

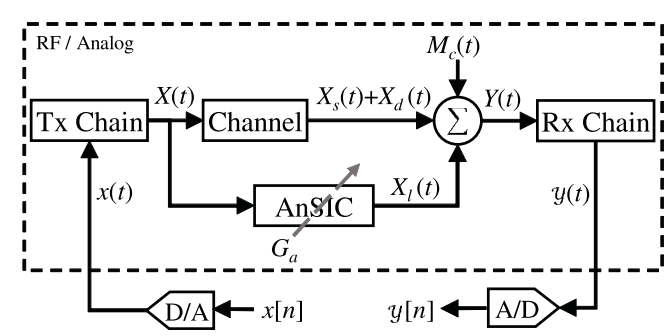

Fig. 3. System model, RF, and analog. The received signal $Y(t)$ includes the Tx environmental reflections while the AnSIC attenuates the direct SI component at the RF front-end by a factor $G_{a}$.

From (1) and (2), it can be shown that the impedance balance between $Z_{\text {ant }}$ and $Z_{\text {bal }}$ causes two equivalent copies of the transmit signal that induce to the secondary winding of the hybrid transformer in opposite phase. Theoretically, this mechanism leads to an infinite direct SI rejection [22].

The balance condition, however, is sensitive to the near-field dynamics as they influence the antenna's impedance. Thus, to maintain the Tx-Rx isolation, an adaptive algorithm is needed to track the substantial environmental changes iteratively and tune $Z_{\text {bal }}$ accordingly.

\section{RADAR-CAPABLE IBFD WIRELESS DEVICE}

By employing the IBFD technology, the Tx and Rx can operate simultaneously on the same channel, which means that the environmental reflections can be recaptured and exploited for remote sensing. From the radar basics, the product of the conjugated transmit signal and the echo from the mobile target yields the Doppler information. However, the received signal in an IBFD communication scheme suffers from two primary sources of interference: first, the distortion caused by a second party transceiver; and second, the direct transmitter leakage.

This section develops the system model for realistic contexts, where there are the above-mentioned interfering signals inherent in an IBFD link. First, we describe the RF and analog bandpass signal model. The model is then extended to the digital baseband by representing Doppler radar signal processing in an ideal case, where there is no interference. Then, we explain how the proposed system overcomes the two distortions introduced above.

\section{A. System Model, RF, and Analog}

Fig. 3 depicts the analog subsystem of the proposed RadCom device. In this scheme, we adopt capital letters to represent the time-domain RF/analog signals whose baseband equivalents are denoted by the corresponding lowercase letters.

In this model, $x(t)$ is the baseband complex-valued information signal, which is indicated by the bandpass communication waveform $X(t)$ after RF upconversion

$$
X(t)=\Re\left\{x(t) e^{j w_{c} t}\right\}
$$

where $\Re\{$.$\} denotes the real part of \{$.$\} and w_{c}(\mathrm{rad} / \mathrm{s})$ is the frequency of carrier wave. 
The static and dynamic environmental reflections of $X(t)$ are also shown by $X_{s}(t)$ and $X_{d}(t)$, respectively, and the notation $X_{l}(t)$ stands for the remaining direct Tx-Rx leakage after the AnSIC block. The desired message arriving at the antenna of the RadCom device is also denoted by $M_{c}(t)$.

As shown in the block diagram in Fig. 3, the received RF signal $Y(t)$ can be expressed as an aggregation of the introduced signals above, i.e.,

$$
Y(t)=X_{s}(t)+X_{d}(t)+M_{c}(t)+X_{l}(t) .
$$

Employing the channel model in [23], we describe the static reflection $X_{s}(t)$ as a sum of the echoed signals through a static multipath channel in the form

$$
X_{s}(t)=\Re\left\{\sum_{s \in P_{s}} A_{s} e^{j \varphi_{s}} x\left(t-\tau_{s}\right) e^{j w_{c}\left(t-\tau_{s}\right)}\right\}
$$

with $P_{s}$ being a set of dominant reflection paths caused by the static objects in the surroundings. $A_{s}$ represents the attenuation factor for the sth path with the initial phase offset $\varphi_{s}$ and propagation delay of $\tau_{s}$.

To simplify the model, we assume that there is a single dynamic object in the environment whose acceleration and path loss time variation are negligible during Doppler estimation. Accordingly, the reflected SI component through the moving object can be written as follows:

$$
X_{d}(t)=\Re\left\{A_{d} e^{j w_{d} t} x\left(t-\tau_{d}\right) e^{j w_{c}\left(t-\tau_{d}\right)}\right\}
$$

where $A_{d}$ denotes the attenuation factor of the dynamic path, and $w_{d}$ determines the frequency shift, which is linearly influenced by the target velocity. $\tau_{d}$ also denotes the propagation delay of the dynamic path.

Similar to the transmit signal from the RadCom device, the received intended message $M_{c}(t)$ can be represented as

$$
M_{c}(t)=M(t) * H_{c}(t) \text { and } M(t)=\Re\left\{m(t) e^{j w_{c} t}\right\}
$$

where $m(t)$ is the baseband complex-valued message transmitted by the remote node TRx, $M(t)$ is the RF representation of $m(t), H_{c}(t)$ characterizes the medium between the two communication devices, and $(*)$ is the time-domain convolution operator.

The residual direct Tx leakage after analog SI suppression also can be expressed as

$$
X_{l}(t)=G_{a} X(t)
$$

where $G_{a}$ stands for the attenuation factor defining the AnSIC's performance to suppress the direct SI component. Note that the on-chip implementation of the AnSIC block in the presented system allows assuming an insignificant time delay between $X_{l}(t)$ and $X(t)$.

Finally, $y(t)$ in Fig. 3 represents the complex-valued baseband received signal after RF downconversion. Given that the communication signal is bandlimited to $B(H z)$, the complex-valued discrete-time representation of $y(t)$ after baseband ADC is

$$
y[n]=y\left(t=\frac{n}{f_{s}}\right) \text { and } f_{s} \geq B
$$

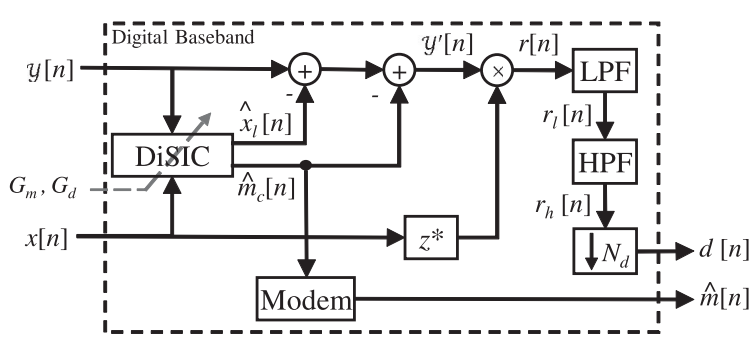

Fig. 4. System model and digital baseband. While the DiSIC block is necessary for in-band bidirectional communication, it can also be employed to suppress the radar-related interference.

with $f_{s}$ being the baseband sampling frequency and the integer $n$ denotes the discrete sample index.

\section{B. Interference-Free Doppler Detection}

Fig. 4 illustrates the digital part of the presented RadCom system. This section explains how the Doppler signal can be extracted from the environmental reflections of the modulated communication signal, while there is no interference from the direct SI leakage and no concurrent transmission by a second party node.

Let define the complex baseband information signal $x[n]$ in terms of time-varying magnitude and phase, i.e.,

$$
x[n]=A_{x}[n] e^{j \varphi_{x}[n]}
$$

where $A_{x}[n]$ and $\varphi_{x}[n]$ determine the amplitude and phase modulations, respectively.

The ideal baseband received signal in the absence of the interfering sources can be defined as

$$
y_{o}[n]=x_{s}[n]+x_{d}[n]
$$

where $x_{s}[n]$ and $x_{d}[n]$ are the static and dynamic environmental reflections, respectively, both are represented in the sampled baseband. Subsequently, using (5) and (6), they can be written in the form

$$
x_{s}[n]=\sum_{s \in P_{s}} A_{s} e^{j \varphi_{s}} x\left[n-n_{s}\right] \text { and } n_{s}=\left\lfloor\tau_{s} f_{s}\right\rfloor
$$

and

$$
x_{d}[n]=A_{d} e^{j w_{d} n} x\left[n-n_{d}\right] \text { and } n_{d}=\left\lfloor\tau_{d} f_{s}\right\rfloor
$$

where $\lfloor\{\}$.$\rfloor is the greatest integer less than or equal to \{$.$\} .$

In the next step, $y_{o}[n]$ is correlated with the conjugated transmit signal $x[n]$. In the context of the RadCom system, this operation reduces the impact of the phase modulation of the communication signal, i.e., $\varphi_{x}[n]$. Employing the definitions in (10)-(13), we define the interference-free radar signal as

$$
\begin{aligned}
r[n] & =y_{o}[n] x^{*}[n]=\left(x_{s}[n]+x_{d}[n]\right) x^{*}[n] \\
& =\eta_{s}[n]+A_{d} e^{j w_{d} n} x\left[n-n_{d}\right] x^{*}[n]
\end{aligned}
$$

where (.)* is the complex conjugate of (.), shown by $z^{*}$ in Fig. 4, and $\eta_{s}[n]$ is the distortion caused by the static reflections, which 
can be derived to be

$$
\eta_{s}[n]=\sum_{s \in P_{s}} A_{s} e^{j \varphi_{s}} x\left[n-n_{s}\right] x^{*}[n] .
$$

In practice, the required sample rate for communication is an order of magnitude larger than the highest Doppler frequency. For example, the maximum velocity in hand gesture detection and vehicular applications ranges from $1 \mathrm{~m} / \mathrm{s}$ up to $200 \mathrm{Km} / \mathrm{h}$, which causes a 16 to $890 \mathrm{~Hz}$ Doppler frequency shift to the traditional 2.4-GHz Wi-Fi signal. This implies that the Doppler information lies in a small portion of the communication bandwidth. To enhance the radar sensitivity, thus, the signal $r[n]$ has to be severely decimated.

A narrow low-pass filter (LPF) can be efficiently realized in practice by a moving average filter. Besides, by exploiting the ergodic property of communication signals, it can be shown that the arithmetic average approximates the expected value [24]. Hence, via employing a moving average filter (shown by the LPF block in Fig. 4), we derive the low-pass filtered radar signal in the form

$$
\begin{aligned}
r_{l}[n] & =\hat{\mathbb{E}}\{r[n]\} \\
& =\hat{\mathbb{E}}\left\{\eta_{s}[n]\right\}+\hat{\mathbb{E}}\left\{A_{d} e^{j w_{d} n} x\left[n-n_{d}\right] x^{*}[n]\right\}
\end{aligned}
$$

where $\hat{\mathbb{E}}\{$.$\} determines the estimated expected value of \{$.$\} ,$ which can be computed over $N_{f}$ successive realizations of the signal as

$$
\hat{\mathbb{E}}\{\cdot\}=\frac{1}{N_{f}} \sum_{N_{f}}\{\cdot\} .
$$

Typically, it is desirable to remove the strong dc component of the radar signal until the nonzero Dopplers can be easily visualized in the ultimate Doppler profile. To this end, our model exploits a dc-blocker filter $h_{h}[n]$, shown by the high-pass filter (HPF) block in the diagram, to suppress the influence of the static reflections in (16), i.e., the term $\hat{\mathbb{E}}\left\{\eta_{s}[n]\right\}$. Given that the target motion is statistically independent of the term $x\left[n-n_{d}\right] x^{*}[n]$, and the Doppler component is in the passband of the LPF, we derive the dc-removed radar signal $r_{h}[n]$ in the form

$$
\begin{aligned}
r_{h}[n]=r_{l}[n] * h_{h}[n] & =\hat{\mathbb{E}}\left\{A_{d} e^{j w_{d} n} x\left[n-n_{d}\right] x^{*}[n]\right\} \\
& =A_{d} \hat{\mathbb{E}}\left\{x\left[n-n_{d}\right] x^{*}[n]\right\} e^{j w_{d} n} .
\end{aligned}
$$

Note that for the moving objects in the range $R<\frac{c}{2 B}$ ( $c$ is the speed of the light in $(\mathrm{m} / \mathrm{s}))$, where $\hat{\mathbb{E}}\left\{x\left[n-n_{d}\right] x^{*}[n]\right\} \approx$ $\hat{\mathbb{E}}\left\{x[n] x^{*}[n]\right\}$, one can approximate $r_{h}[n]$ as

$$
r_{h}[n] \simeq A_{d} \hat{P}_{x} e^{j w_{d} n}
$$

where the term $\hat{P}_{x}=\hat{\mathbb{E}}\left\{A_{x}^{2}[n]\right\}$ denotes the estimated power of the transmit signal $x[n]$.

To accomplish the decimation and to facilitate frequency analysis, the dc-removed radar signal $r_{h}[n]$ is downsampled to obtain the narrow band Doppler signal $d[n]$ as

$$
d[n]=r_{h}\left[n N_{d}\right]
$$

where given the baseband sample rate $f_{s}$ and the decimation factor $N_{d}, d[n]$ streams at $f_{s} / N_{d}$.
Using fast Fourier transform (FFT), we can analyze $d[n]$ to yield its dominant frequency component, i.e., the Doppler frequency $f_{d}=\frac{w_{d}}{2 \pi}$, and the instantaneous radial target velocity (relative to the radar) $\mathrm{v} \mathrm{m} / \mathrm{s}$ )

$$
f_{d}=2 \frac{v}{c} f_{c}
$$

where $f_{c}=\frac{w_{c}}{2 \pi}$ is the carrier frequency in Hz. Subsequently, the velocity resolution $v_{\text {res }}$ and the maximum detectable velocity $v_{\text {max }}$ can be determined as follows:

$$
\begin{aligned}
v_{\text {res }} & =\frac{1}{2} \frac{c}{f_{c}} \frac{f_{s}}{N_{d} N_{\mathrm{fft}}} \\
|v|<v_{\max } & =\frac{1}{4} \frac{c}{f_{c}} \frac{f_{s}}{N_{d}}
\end{aligned}
$$

with $N_{\text {fft }}$ being the number of samples used to obtain the FFT, and $|$.$| denotes the absolute value of \{$.$\} .$

It is worthwhile to mention that, regarding the application conditions discussed earlier, the system is also applicable to the multicarrier waveforms, e.g., orthogonal frequency-division multiplexing (OFDM), as the imposed Doppler shifts to the orthogonal subcarriers are nearly identical in such a way that the resultant Doppler signal is a superposition of multiple cofrequency Dopplers.

\section{Doppler Extraction in the Presence of Distortion Sources}

As described earlier, in an IBFD scenario, the received signal also includes the interference signals

$$
y[n]=y_{o}[n]+m_{c}[n]+x_{l}[n]
$$

where $y_{o}[n]$ is the interference-free received signal introduced in (11), $m_{c}[n]=M_{c}\left(t=\frac{n}{f_{s}}\right)$ denotes the received intended message in the digital baseband, and $x_{l}[n]$ stands for the residual Tx leakage, which can be defined according to its analog equivalent in (8) as

$$
x_{l}[n]=X_{l}\left(t=\frac{n}{f_{s}}\right)=G_{a} x[n] .
$$

While the DiSIC block is necessary for communication, the proposed system also applies it to enhance the radar. As shown in Fig. 4, the estimated residual direct SI $\hat{x}_{l}[n]$ and the estimated message $\hat{m}_{c}[n]$ are subtracted from the interfered received signal $y[n]$ defined in (24). Accordingly, the enhanced received signal $y^{\prime}[n]$ (see Fig. 4) can be derived to be

$$
\begin{aligned}
y^{\prime}[n] & =y[n]-\left\{\hat{m}_{c}[n]+\hat{x}_{l}[n]\right\} \\
& =y_{o}[n]+m_{c}[n]-\hat{m}_{c}[n]+x_{l}[n]-\hat{x}_{l}[n] \\
& =y_{o}[n]+G_{m} m_{c}[n]+G_{d} x_{l}[n]
\end{aligned}
$$

where $G_{m}$ determines the performance of DiSIC to attenuate the interference caused by the received desired message $m_{c}[n]$, and $G_{d}$ characterizes the DiSIC's capability to reduce the remaining direct SI leakage $x_{l}[n]$. Note that the estimated signal $\hat{m}_{c}[n]$ is also fed into the communication modem to extract the message $\hat{m}[n]$.

The interfered radar signal then can be defined as

$$
r[n]=y^{\prime}[n] x^{*}[n] .
$$


To aid our derivations and to facilitate the analysis of the distortions, we rewrite the nonideal low-pass filtered radar signal $r_{l}[n]$ in terms of the two introduced interferences and the Doppler information as follows:

$$
\begin{aligned}
r_{l}[n] & =\hat{\mathbb{E}}\{r[n]\} \\
& =\hat{\mathbb{E}}\left\{\left(y_{o}[n]+G_{m} m_{c}[n]+G_{d} x_{l}[n]\right) x^{*}[n]\right\} \\
& =\hat{\mathbb{E}}\left\{y_{o}[n] x^{*}[n]\right\}+\hat{\mathbb{E}}\left\{\eta_{m}[n]\right\}+\hat{\mathbb{E}}\left\{\eta_{l}[n]\right\}
\end{aligned}
$$

where $\eta_{m}[n]$ denotes the distortion produced by the received desired message $m_{c}[n], \eta_{l}[n]$ represents the distortion introduced by the residual direct SI leakage $x_{l}[n]$, and the term $\hat{\mathbb{E}}\left\{y_{o}[n] x^{*}[n]\right\}$ comprises the Doppler information as explained in the interference-free case in the previous part.

\section{Distortion Analysis}

1) Interference From the Desired Received Message $\eta_{m}[n]$ : as shown in (26), by minimizing $G_{m}$, the DiSIC block can suppress the impact of a probable concurrent in-band transmission on the radar signal.

More importantly, in a real-world IBFD communication scheme, the two transmit signals are likely orthogonal, i.e., $\mathbb{E}\left\{m_{c}[n] x^{*}[n]\right\}=0$. This allows to approximate the filtered $\eta_{m}[n]$ from (28) as

$$
\hat{\mathbb{E}}\left\{\eta_{m}[n]\right\}=G_{m} \hat{\mathbb{E}}\left\{m_{c}[n] x^{*}[n]\right\} \approx 0 .
$$

2) Distortion From the Remaining Direct Tx Leakage $\eta_{l}[n]$ : regarding the equations (10), (25) and (28), the interference from the residual SI can be derived to be

$$
\begin{aligned}
\hat{\mathbb{E}}\left\{\eta_{l}[n]\right\} & =\hat{\mathbb{E}}\left\{G_{d} x_{l}[n] x^{*}[n]\right\} \\
& =\hat{\mathbb{E}}\left\{G_{a} G_{d} x[n] x^{*}[n]\right\}=G_{l} \hat{P}_{x}
\end{aligned}
$$

where $G_{l}=G_{a} G_{d}$ is the joint analog and digital direct Tx leakage rejection.

Similar to the dc term caused by the static reflection in (16), it is already clear that the dc-removal filter will also suppress the distortion $\eta_{l}[n]$. However, further attenuation is required as the direct Tx leakage is orders of magnitude stronger than the reflected signal from the target, i.e., $x_{d}[n]$. As shown in (30), the analog and digital SI cancellers directly influence this distortion in such a way that by improving the direct $\mathrm{Tx}-\mathrm{Rx}$ isolation, i.e., $G_{l} \rightarrow 0$, the RadCom system can effectively lessen $\eta_{l}[n]$, without affecting the environmental reflections which are needed for Doppler extraction.

\section{E. Minimum Detectable Velocity as a Function of AnSIC Tuning Rate}

In the nonideal SI cancelation scheme, the EBD has to be re-tuned frequently to maintain sufficient analog SI rejection. Practically, triggering the adaptation algorithm is followed by a random phase shift to the received signal. The EBD tuning rate $f_{\text {tune }}$ hence limits the minimum detectable velocity $v_{\min }$ as the EBD should be stable during the segment used for deriving the Doppler spectrum. Therefore, the duration of the segment, determined by the sample rate, decimation factor and FFT size should be limited which means $f_{\text {tune }} \leq \frac{f_{s}}{N_{d} N_{\text {fft }}}$. Combining this
TABLE I

IEEE 802.11P-LIKE OFDM WAVEFORM CHARACTERISTICS

\begin{tabular}{|c|c||c|c|}
\hline Param. & Value & Param. & Value \\
\hline strength & $20 \mathrm{dBm}$ & OFDM subcarriers & 52 \\
\hline bandwidth & $10 \mathrm{MHz}$ & OFDM pilots & 4 \\
\hline bit rate & $6 \mathrm{Mbps}$ & modulation & QPSK \\
\hline
\end{tabular}

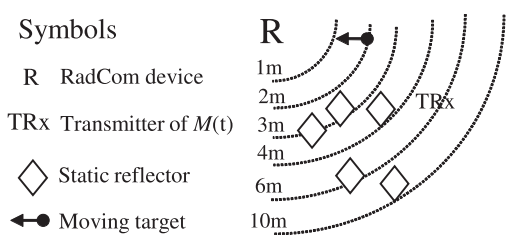

Fig. 5. Layout of the simulated multipath channel.

with (22) we can define the minimum velocity that remains detectable, i.e.,

$$
|v| \geq v_{\min }=\frac{1}{2} \frac{c}{f_{c}} f_{\text {tune }} .
$$

\section{Simulation Results}

In this section, we simulate the proposed RadCom system in the presence of the interfering sources. The simulation aims to

1) evaluate the impact of SI cancelation on the radar'

2) investigate the radar performance in an IBFD communication scenario;

3) study the effectiveness of using the DiSIC module to remove the interference caused by a second party transmitter.

To this end, we chose an OFDM waveform for both the selftransmit signal $x[n]$ and the message $m[n]$, applying parameters as specified in Table I for IEEE 802.11p. Fig. 5 illustrates the simulated scenario where five motionless reflectors are positioned within 3-10 $\mathrm{m}$ around the radar-enabled IBFD device to resemble an indoor multipath channel. A mobile target is also involved in the simulation to produce the Doppler frequency shift, and the transceiver TRx is located at $6 \mathrm{~m}$.

The simulation is carried out by MATLAB in double precision floating point; the in-band thermal noise is assumed to be $-90 \mathrm{dBm}$ and the IBFD receiver samples at $40 \mathrm{MHz}$. The transmit signals $(x[n]$ and $m[n])$ are modulated by two statistically independent and zero-mean random data streams. To obtain the interfered radar signal defined in (28), the simulator computes the baseband received signal $y[n]$ taking into account the defined terms in (12), (13), and (25). The decimated and dc-removed Doppler signal $d[n]$ is then estimated at $610 \mathrm{~Hz}$ (decimation factor $N_{d}=2^{16}$ ), and windowed (Hanning) to be analyzed by FFT. Assuming an observation window of $1000 \mathrm{~ms}$ for a transmit signal at 2.4-GHz RF band, this configuration enables up to $18.42 \mathrm{~m} / \mathrm{s}$ speed measurement with velocity resolution of $6.04 \mathrm{~cm} / \mathrm{s}$.

Fig. 6 shows the power spectrum of the low-pass filtered and downsampled components of the radar signal in (28), without applying the dc-removal filter. In this test, the total SI rejection is set to be $G_{l}=-40 \mathrm{~dB}$, and a target in $2 \mathrm{~m}$ from the radar moves at $5 \mathrm{~m} / \mathrm{s}$. Note that despite $40-\mathrm{dB} \mathrm{Tx}-\mathrm{Rx}$ isolation, the distortion $\eta_{l}[n]$ is still the dominant interference. In this graph, 


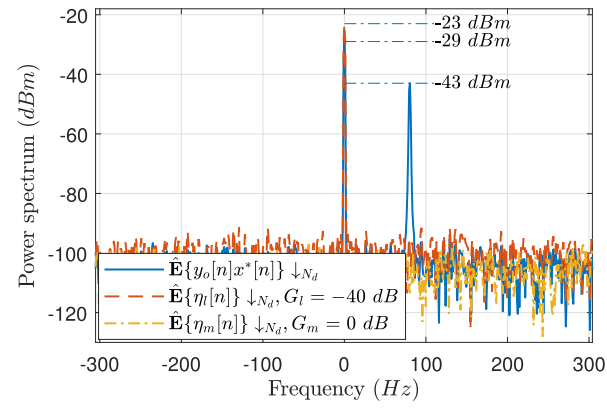

Fig. 6. Power spectrum of the decimated (low-pass filtered and downsampled by the factor $N_{d}=2^{16}$ ) components in (28) without dc removing, including the distortion of the direct SI $\eta_{l}[n]$, the interference from the desired communication message $\eta_{m}[n]$, and the ideal radar signal, i.e., $y_{o}[n] x^{*}[n]$.

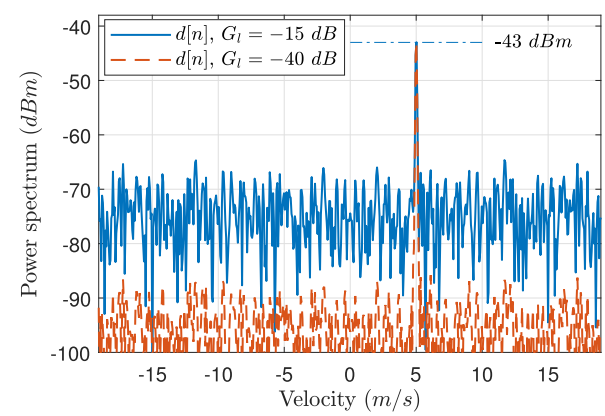

Fig. 7. Power spectrum of the estimated Doppler signal $d[n]$ versus velocity $(\mathrm{m} / \mathrm{s})$ as a function of the total Tx leakage rejection $G_{l}$, assuming the target is located at $d_{t}=2 \mathrm{~m}$ from the IBFD device and moves at $v=5 \mathrm{~m} / \mathrm{s}$.

the nonzero component at $81.4 \mathrm{~Hz}$ indicates the Doppler frequency shift, which is relatively less than the dc components caused by the static reflections and the residual direct leakage $x_{l}[n]$. Furthermore, note that the distortion caused by TRx, i.e., the term $\eta_{m}[n]$, is as weak as the noise level, even though there is no compensation by the DiSIC module $\left(G_{m}=0 \mathrm{~dB}\right)$.

\section{A. Radar Performance Evaluation}

In the next step, we repeat the test for 15- and 40-dB direct SI isolation to evaluate the impact of the direct Tx leakage. This time, the enhanced received signal $y^{\prime}[n]$ in (26) is feed to the radar mixer. The resultant Doppler signal, after low-pass filtering, dc removing, and downsampling is also shown in Fig. 7. Note that in this graph, the frequency axis ( $x$-axis) is mapped to velocity by applying the definition in (21). As shown, decreasing $G_{l}$ improves the SNR of the detected Doppler signal as it significantly suppresses the impact of the direct Tx leakage. Furthermore, the graph in Fig. 7 shows a $-43 \mathrm{dBm}$ Doppler component at $5(\mathrm{~m} / \mathrm{s})$ for both SI rejection levels.

Regarding the simulation results mentioned above, we adopt the SNR of the resultant Doppler signal $\mathrm{SNR}_{d}$ as a metric to assess the radar performance. To this end, the power of the Doppler component (relative to the noise floor) is computed in the range of $\pm 2 v_{\text {res }}$ around the expected target velocity.

Fig. 8 depicts the estimated $\mathrm{SNR}_{d}$ for different levels of Tx leakage rejection. From this figure, it is obvious that decreasing $G_{l}$ improves the quality of the Doppler signal in all cases. Moreover, the results reveal that the system offers higher performance

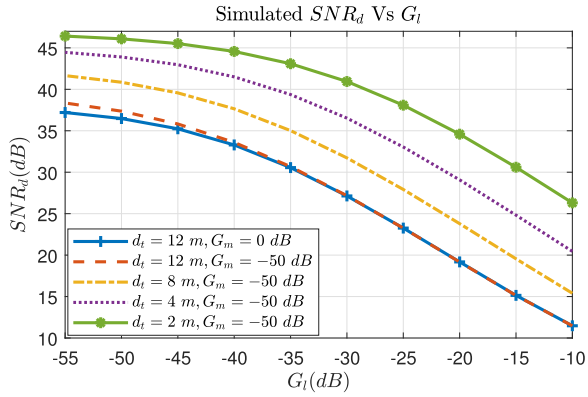

Fig. 8. Simulated SNR of the Doppler signal $\left(\mathrm{SNR}_{d}\right)$ versus the joint analog and digital Tx leakage rejection $G_{l}$, as a function of the distance between the target and the RadCom device $\left(d_{t}\right)$, with and without compensation for the interfering message $m_{c}[n]$, i.e., $G_{m}=-50 \mathrm{~dB}$ and $G_{m}=0 \mathrm{~dB}$.
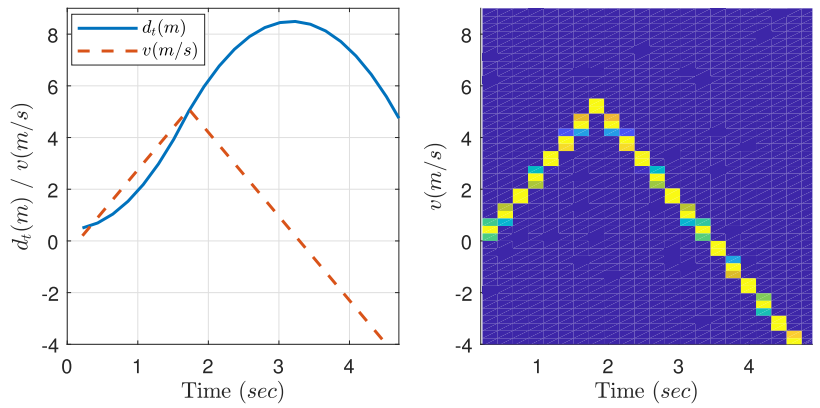

Fig. 9. (Left) Distance and the radial speed of the simulated target, i.e., $d_{t}$ and $v$, respectively. (Right) Estimated Doppler profile of a target moving in a simulated scenario.

as the target approaches the IBFD radio and the system captures stronger reflections.

As explained in the system model representation, the orthogonality of the transmit signals nullifies the distortion caused by a concurrent transmission. Still, it is interesting to assess the effectiveness of the digital cancelation of the intended message on radar performance. Fig. 8 also shows the performance when the DiSIC module provides no compensation $\left(G_{m}=0 \mathrm{~dB}\right)$ for the received message $m_{c}[n]$ (the case with $d_{t}=12 \mathrm{~m}$ ). This proves that given $x[n]$ and $m_{c}[n]$ are indeed orthogonal, the interference from the desired signal is insignificant on the Doppler estimation performance.

\section{B. Doppler Profile}

The simulation proceeds by moving the target at different velocities. As illustrated in Fig. 9 (left), the target first moves away from the device at constant acceleration. At $4.4 \mathrm{~m}$, it decreases the speed until it stops at $8.5 \mathrm{~m}$ and returns toward the device with the same acceleration. The Tx-Rx direct isolation is set to $50 \mathrm{~dB}$ to provide constant SI rejection along with the simulation. Fig. 9 (Right) illustrates the estimated Doppler profile, which precisely follows the ground-truth curve at the left.

\section{RADAR-CAPABLE IBFD PROTOTYPE}

Fig. 10 (left) illustrates the functional schematic of the prototyped device whose picture is shown in Fig. 10 (right). To the best of our knowledge, it is the first demonstration of EBD-based monostatic Doppler radar, which is integrated into 


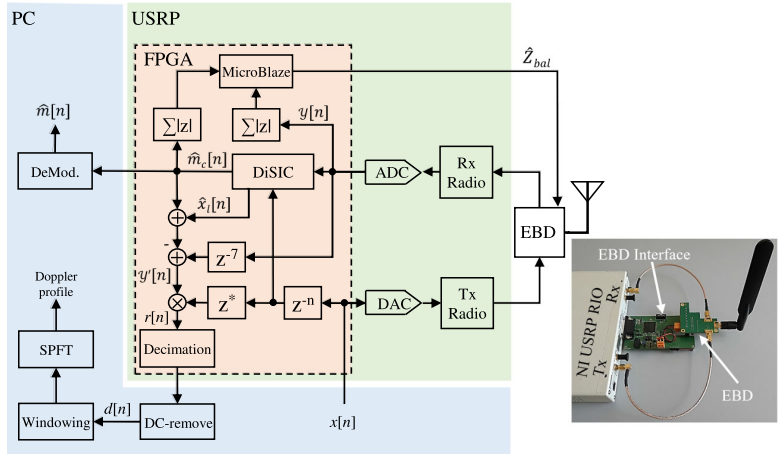

Fig. 10. Block diagram (left) and the picture (right) of the prototyped RadCom system.

an IBFD platform and enables simultaneous communication and opportunistic wireless environment sensing.

The implemented system consists of one SDR, one customized EBD-based AnSIC module [19], and one PC. The SDR is an NI USRP RIO with a Kintex-7 FPGA onboard. The EBD is interfaced with the USRP by two $25-\mathrm{cm}$ RF cables and a GPIO bus. The RF cables connect the EBD and the USRP's radio front-end. The whole setup can be controlled and monitored via a PCI-e connection and a LabVIEW user interface. Regarding the EBD's maximum tolerable RF input and its operating frequency band, the power of the OFDM signal is limited to $-8 \mathrm{dBm}$ and the USRP's RF front end is tuned to $1.74 \mathrm{GHz}$. We also use an omnidirectional antenna as such the device can radiate/receive to/from all directions and maintain its communication functionality. To synchronize the Tx-baseband to Rx-baseband signals, the model employs a $n$-tap delay block shown by $z^{-n}$ in the block diagram. By connecting the EBD's antenna port to a $50-\Omega$ dummy load, we measured the delay $n=119$ samples in our prototype. The USRP produces $60-\mathrm{MHz}$ complex baseband signal which has to be mixed with the transmitted signal. Prior to the mixer, the estimated signals $\hat{m}_{c}[n]$ and $\hat{x}_{l}[n]$ are subtracted from the received signal. A seven-sample delay block is also embedded to compensate for the latency with the digital SI canceller. The mixed signal is then decimated to achieve narrow-band complex signal with a configurable rate in the range of 457 to $1828 \mathrm{~Hz}$. The real-time processing on the FPGA is in 16-b and 32-b fixed-point precision and, as shown in Fig. 10, the rest of the computation is accomplished on a computer to build up a two-dimensional (2-D) Doppler profile. Theoretically, this configuration enables detecting targets moving up to $78.73 \mathrm{~m} / \mathrm{s}$ with $8.6 \mathrm{~cm} / \mathrm{s}$ speed resolution (for 1 -s observation time).

\section{A. EBD Tuning Implementation}

The EBD tuner in our system is deployed on the MicroBlaze softcore [25] which enables real-time adaptation within $0.125 \mathrm{~ms}$. As shown in the prototype schematic, the instantaneous SI is observed through feedback from the EBD receiver port. Based on the level of the measured SI, the tuner adjusts the impedance network adaptively, seeking to maximize the Tx-Rx isolation. The $Z_{\text {bal }}$ in the prototyped EBD comprises four adjustable capacitors, each can obtain $2^{8}$ distinct capacitance
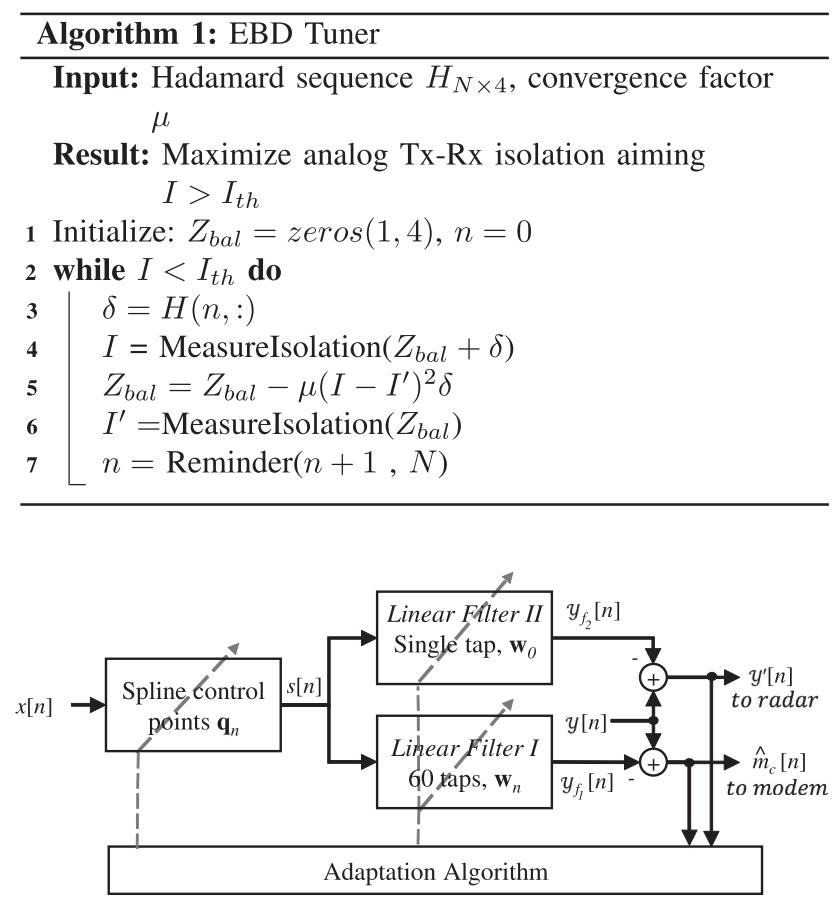

Fig. 11. Adaptive spline-based Hammerstein model used for DiSIC. While the multitap filter can estimate the remaining SI signal, the single-tap filter merely computes the residual direct Tx leakage without affecting the environmental SI echoes.

values. In this article, the dithered linear search (DLS) algorithm [26], presented in Algorithm V-A, is adopted to explore a 4-D solution space, finding the optimum point where the analog SI isolation $I$ is better than a certain threshold $I_{\text {th }}$.

The DLS algorithm is a gradient descent optimizer in which signals with small amplitude and zero-mean, the dither sequences $\delta$, are intentionally injected into the impedance network parameters, i.e., $Z_{\text {bal }}=\left\{c_{1}, c_{2}, c_{3}, c_{4}\right\}$. The additive independent and uncorrelated dither signals allow multiple duplexer parameters can be updated concurrently. The dithers are generated by Hadamard sequences in our implementation.

Once the EBD is initialized, the tuner also measures the level of the estimated $\hat{m}_{c}[n]$ by the DiSIC block. This mechanism guarantees appropriate residual SI measurement in the full-duplex communication mode.

\section{B. Digital SI Canceller Implementation}

On top of analog SI rejection, digital cancelation is also needed to achieve sufficient SNR required for communication as well as to improve the radar performance. The digital SI canceller in this article models both linear and nonlinear impacts of the physical circuitry and the channel. The DiSIC block is based on an adaptive spline-based Hammerstein model consisting of a spline-interpolated lookup table cascaded with an adaptive linear filter [27], [28]. The former model matches the static nonlinearities and the latter tracks the linear part of the SI signal. This configuration closely resembles the physical system and the most influential source of nonlinear distortion, the transmitter power amplifier and the linear SI channel. Fig. 11 illustrates the basic Hammerstein DiSIC mode which is briefly summarized 
below. For a complete description, including derivations and equations, we refer the reader to [28].

As shown in Fig. 11, the baseband transmit signal $x[n]$ is fed to the spline model, which produces the spline estimate $s[n]$ using a lookup table $\mathbf{q}_{n}$ and a second-order spline interpolator.

Then, a 60-tap linear filter, shown by Linear Filter I, produces the cancelation signal $y_{f_{1}}[n]$, that is the estimate of the direct Tx leakage and the environmental SI reflections, i.e., $y_{f_{1}}[n]=\hat{x}_{l}[n]+\hat{x}_{s}[n]+\hat{x}_{d}[n]$. The estimated SI is subtracted from the received signal to yield $\hat{m}_{c}[n]$, which is required to perform communication. While removing the environmental Tx reflections requires an adaptive filter with multiple taps, it can be shown that a single tap filter is sufficient to suppress the residual direct Tx leakage merely. Accordingly, a single tap filter, demonstrated by the Linear Filter II block, is embedded to generate the cancelation signal $y_{f_{2}}[n]=\hat{x}_{l}[n]$.

The DiSIC block exploits a standard least-mean squares optimizer [29] to update the adaptive filter parameters dynamically. This algorithm utilizes the signals $\hat{m}_{c}[n]$ and $y^{\prime}[n]$ as feedback to converge to the optimum state. To achieve real-time functionality, the DiSIC is fully implemented on the FPGA. The 60-tap filter implementation consists of 533 DSP units, each of which can handle up to $25 \times 18$-b multiplications. The same FPGA realization allocates 67632 LUTs. The DiSIC block has seven pipeline stages with seven clock cycles latency and provides up to 40-dB Tx-Rx isolation. In our implementation, Linear Filter $I$ is tuned every eight samples while the system adjusts Linear Filter II at the same rate as the EBD.

\section{EXPERIMENTAL RESULTS}

This section details the experimental results obtained by the system prototype. First, the required EBD tuning rate is measured in an indoor environment. Second, the SI rejection performance is shown, and finally, we assess the accuracy and the performance of the velocity estimation experimentally.

\section{A. EBD Tuning Tradeoff}

In Section II, we discussed the necessity of EBD retuning and introduced $f_{\text {tune }}$ as a parameter that limits the minimum detectable velocity. In this part, we investigate $f_{\text {tune }}$ in an indoor environment. Fig. 12 displays the CDFs of the EBD isolation, measured in a typical office room where the device is placed on a desk next to a working person at $0.5 \mathrm{~m}$. In each experiment, an isolation threshold is specified to trigger the EBD tuning algorithm. The level of the SI signal and the number of triggers are recorded for 60 min to estimate how often the EBD has to be tuned to maintain the $\mathrm{Tx}-\mathrm{Rx}$ isolation below the threshold. Note that the actual achieved isolation depends on the EBD setting and the environment, giving a distribution of instantaneous isolation values. Each time the EBD is retuned, a more optimal configuration for the given environment is determined. It is evident that increasing the isolation threshold raises the required EBD tuning speed and, consequently, increases the minimum detectable velocity expressed by (31). According to the experimental results in Fig. 12, to obtain 50-, 45-, and 40-dB AnSIC, the EBD requires, respectively, 1.3-, 0.93-, and $0.5-\mathrm{Hz}$

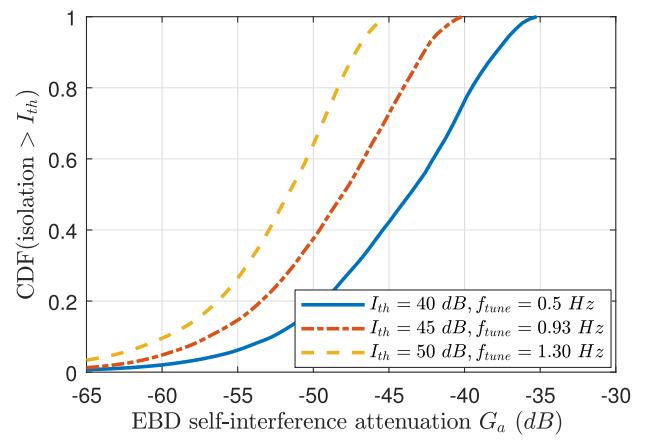

Fig. 12. Empirical cumulative distribution function (CDF) plot of the EBD isolation performance versus various tuning thresholds $I_{\mathrm{th}}$, measured in an indoor environment.

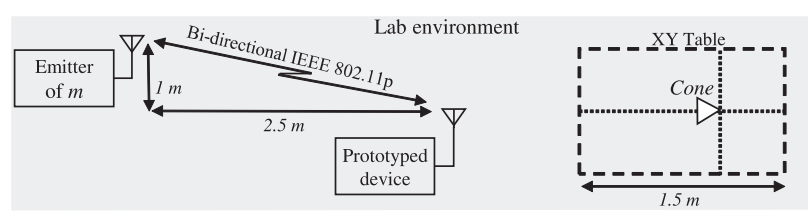

Fig. 13. Schematic of the testbed. Two IBFD devices are used to form an IBFD link, and a XY positioner moves a cone reflector at various velocities.

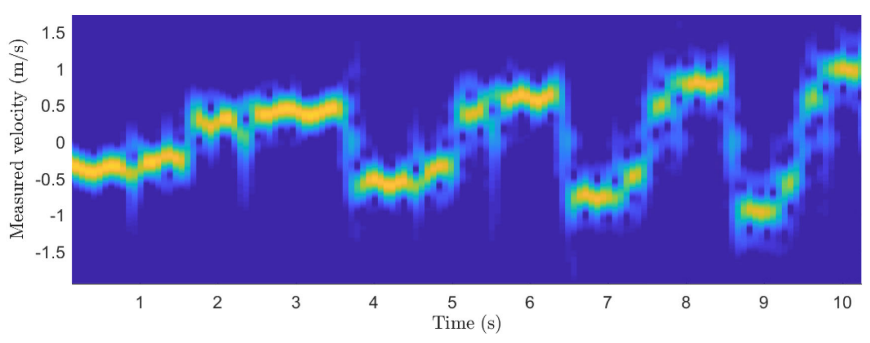

Fig. 14. Measured Doppler profile produced by the prototyped device representing the movement of a mounted cone reflector on a XY table, which moves at $\pm 0.4, \pm 0.6, \pm 0.8$, and $\pm 1 \mathrm{~m} / \mathrm{s}$. Short-time Fourier transform (STFT) segment $500 \mathrm{~ms}$ with $85 \%$ overlap, Doppler signal at $915 \mathrm{~Hz}$, Hanning window, 8K-point FFT, and $G_{l}<-45 \mathrm{~dB}$.

tuning rates. These tunning frequencies limit $v_{\min }$ to $0.11,0.80$, and $0.43 \mathrm{~m} / \mathrm{s}$, respectively.

\section{B. Doppler Measurement}

To evaluate the radar capability of the prototyped device, a remotely controlled XY table is utilized to move a cone reflector with radar cross section $-17 \mathrm{dBsm}$ at different speeds ranging from 0.2 to $1 \mathrm{~m} / \mathrm{s}$, as shown in Fig. 13. Due to the omnidirectionality of the antenna, the system is also exposed to statistic reflections from a lab environment. Another communication device is also placed at $2.7 \mathrm{~m}$ from the prototype to enable IBFD communication. The radios transmit packets starting with IEEE 802.11ac header, followed by a random bitstream.

Fig. 14 shows the Doppler profile obtained by STFT, when the reflector moves forward and backward at different velocities. Each segment in this spectrogram is $500 \mathrm{~ms}$, including $85 \%$ overlap with the adjacent frame. Due to inertia in the mechanical setup, there is a visible artifact in the spectrogram when the target changes the direction. 


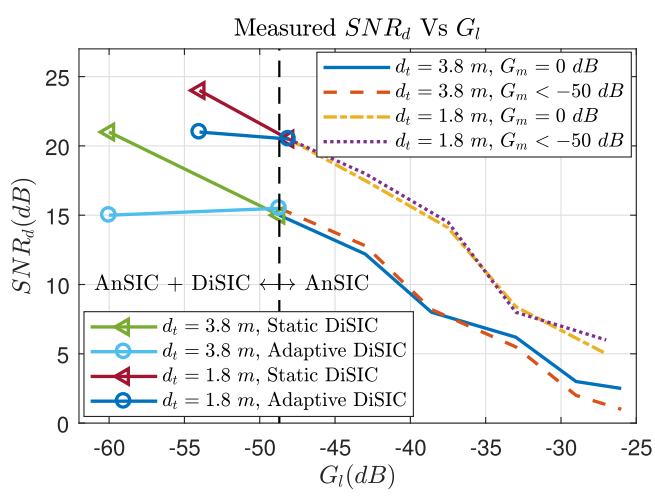

Fig. 15. Measured $\mathrm{SNR}_{d}$ versus the total Tx leakage rejection $G_{l}$ as a function of the target distance from the radar $d_{t}$, when a moving reflector at 1.8 and $3.8 \mathrm{~m}$ moves at $0.3 \mathrm{~m} / \mathrm{s}$ and the radar transmits a $-8 \mathrm{dBm}$ IEEE $802.11 \mathrm{p}$-like waveform at $1.74 \mathrm{GHz}$. The static AnSIC improves the radar performance. Similarly, the single tap filter of the DiSIC module should remain unchanged otherwise it does not enhance the radar sensitivity.

Fig. 15 shows the measured $\mathrm{SNR}_{d}$ when the reflector is located at 1.8 and $3.8 \mathrm{~m}$ from the radar. As indicated by the vertical dashed line in this graph, up to $48-\mathrm{dB} \mathrm{Tx}-\mathrm{Rx}$ isolation is provided only by the AnSIC block. As expected, the measured result in this experiment proves that the static AnSIC by the EBD suppresses the SI sufficiently enough to enable both communication and Doppler detection. Furthermore, better Doppler quality is achievable for the objects closer to the radar. For further Tx-Rx isolation, the DiSIC module is also utilized. This graph also shows that the SI rejection is increased when DiSIC adaptively tunes the single-tap filter. However, this adaptive digital filter does not improve $\mathrm{SNR}_{d}$ as it partially cancels the Doppler as well. We also observed that a filter with eight adaptive taps can thoroughly remove the Doppler. Combining static and dynamic digital filters, thus, improves both communication and radar performance.

To evaluate the impact of the DiSIC compensation for $m_{c}[n]$ on radar performance in the full-duplex mode, the measurement also has been done with and without subtracting the estimated communication signal $\hat{m}_{c}[n]$ from the received signal, i.e., $G_{m}<-50 \mathrm{~dB}$ and $G_{m}=0 \mathrm{~dB}$, respectively. As illustrated in Fig. 15, this experiment verifies that the impact of the interference from another emitter in full-duplex communication mode is insignificant, even when the two transmitted information packets, $x[n]$ and $m_{c}[n]$ in this case for example, carry the same header.

Fig. 16 presents the performance in terms of the probability of correct velocity detection $\left(P_{d}\right)$ and the likelihood of false alarm $\left(P_{\mathrm{fa}}\right)$. For each measurement in this figure, the reflector moves (forward and backward) five times at the speed of $0.2 \mathrm{~m} / \mathrm{s}$. The measurements are performed for various levels of EBD SI cancelation. Then, the spectrogram is formed and the most significant FFT component is chosen to compute the target velocity. Next, the estimated speed is compared with the ground-truth. By changing the detection thresholds, various probabilities are measured. Regarding the results in Fig. 16, increasing the direct SI suppression improves the likelihood of correct velocity detection.

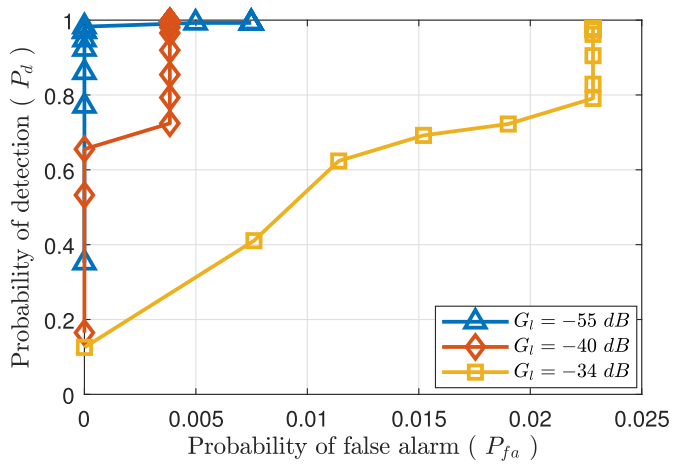

Fig. 16. Measured $P_{d}-P_{f a}$ graph for different levels of direct SI rejection and the target moves at $0.2 \mathrm{~m} / \mathrm{s}$.

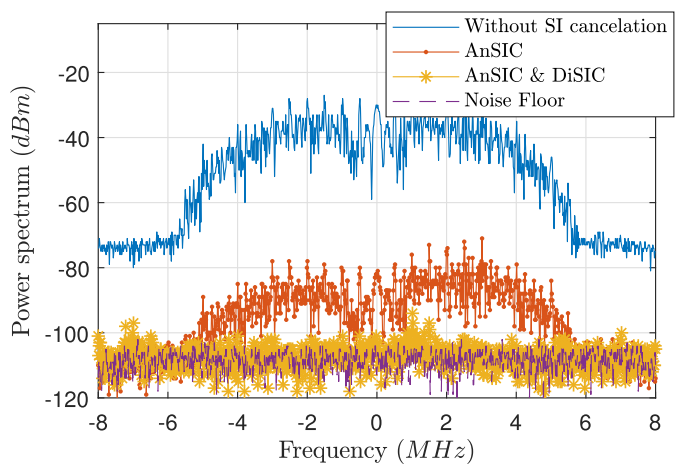

Fig. 17. Measured power spectrum of the received signal, with and without SI cancelation. Joint analog and digital modules are required to enable in-band bidirectional communication.

\section{SI Rejection for Communication}

Fig. 17 demonstrates the measured power spectrum of the received OFDM signal, including the direct and environmental SI components. This graph shows how the combination of AnSIC and DiSIC modules in our prototype can suppress the SI signal to the noise level, allowing bidirectional communication. We measured the average bit error rate of the IBFD link $<1.5 \%$ at both sides in the testbed shown in Fig. 13.

\section{DISCUSSION}

In contrast with the classic monostatic radars, there are some constraints imposed by the simultaneous communication functionality of the proposed RadCom system. First of all, the radar relies on the Tx communication signal, and, hence, cannot sense when the Tx is idle. One approach to resolve it is to simply interpolate between the sensing periods. Another possible approach is to add a bistatic radar functionality to also benefit from the presence of other Tx signals. The majority of the required hardware is already available for that purpose.

Besides, since the communication standards primarily dictate the operation bandwidth, it seems inefficient to add the range detection functionality for low-bandwidth communication links. Furthermore, to decode a low-SNR communication signal in a highly dynamic environment, the system has to increase the tuning rate of the analog SI canceller. Consequently, it restricts the 
minimum detectable velocity of the radar since the observation time is reduced in that case.

\section{CONCLUSION}

This article presents an IBFD RadCom system that enables joint communication and opportunistic wireless sensing, and to the author's knowledge, it is the first of its kind. Our solution can be built into future IBFD devices without extra hardware deployment. In this article, we study how various interfering sources influence the performance of the radar. The proposed system is evaluated using an IEEE 802.11p-like waveform and a prototyped device, consisting of an EBD-equipped SDR. The implemented digital SI canceller benefits from a 60-tap adaptive filter, which, jointly with the EBD, delivers $>85-\mathrm{dB}$ SI rejection required for communication. A single tap digital filter that has to be tuned regarding the EBD's adaptation rate is also utilized to enhance the radar signal further. We conclude that this adaptation rate limits the minimum detectable velocity. The proof-of-concept prototyped system in this article can precisely measure velocities in the range of $0.2-1 \mathrm{~m} / \mathrm{s}$ while the device establishes an in-band bidirectional link with another IBFD device.

According to the simulation and experimental results, the radar performance is mainly dependent on the extent of direct SI cancelation, whereas, in the full-duplex mode, the distortion caused by an adjacent communication device is negligible as long as both transmit signals are uncorrelated.

More work is needed to derive system specifications, in terms of system bandwidth, symbol, and packet length, that are suitable both for radar and communication systems. Moreover, in the future, the proposed RadCom system could be equipped with an antenna array to further estimate the signal direction of arrival.

\section{REFERENCES}

[1] X. Zhu and Y. Feng, "RSSI-based algorithm for indoor localization," Commun. Netw., vol. 5, no. 2, pp. 37-42, 2013.

[2] X. Luo, W. J. O'Brien, and C. L. Julien, "Comparative evaluation of received signal-strength index (RSSI) based indoor localization techniques for construction jobsites," Adv. Eng. Inform., vol. 25, no. 2, pp. 355-363, 2011.

[3] H. Abdelnasser, M. Youssef, and K. A. Harras, "WiGest: A ubiquitous WiFi-based gesture recognition system," in Proc. IEEE Conf. Comput. Commun., 2015, pp. 1472-1480.

[4] S. Sigg, U. Blanke, and G. Troster, "The telepathic phone: Frictionless activity recognition from WIFI-RSSI," in Proc. IEEE Int. Conf. Pervasive Comput. Commun., 2014, pp. 148-155.

[5] L. Sun, S. Sen, D. Koutsonikolas, and K.-H. Kim, "Widraw: Enabling hands-free drawing in the air on commodity WIFI devices," in Proc. 21 st Annu. Int. Conf. Mobile Comput. Netw., 2015, pp. 77-89.

[6] B. Kellogg, V. Talla, and S. Gollakota, "Bringing gesture recognition to all devices," in Proc. 11th USENIX Conf. Netw. Syst. Des. Implementation, 2014, vol. 14, pp. 303-316.

[7] K. Wu, J. Xiao, Y. Yi, M. Gao, and L. M. Ni, "FILA: Fine-grained indoor localization," in Proc. IEEE INFOCOM, 2012, pp. 2210-2218.

[8] Q. Pu, S. Gupta, S. Gollakota, and S. Patel, "Whole-home gesture recognition using wireless signals," in Proc. 19th Annu. Int. Conf. Mobile Comput. Netw., 2013, pp. 27-38.

[9] B. Tan, K. Woodbridge, and K. Chetty, "A real-time high resolution passive WiFi Doppler-radar and its applications," in Proc. Int. Radar Conf., 2014, pp. 1-6.

[10] W. Li, B. Tan, R. J. Piechocki, and I. Craddock, "Opportunistic physical activity monitoring via passive WiFi radar," in Proc. IEEE 18th Int. Conf. e-Health Netw., Appl. Serv., 2016, pp. 1-6.
[11] W. Li, B. Tan, and R. J. Piechocki, "Non-contact breathing detection using passive radar," in Proc. IEEE Int. Conf. Commun., 2016, pp. 1-6.

[12] B. Tan and K. Woodbridge, "A wireless passive radar system for real-time through-wall movement detection," IEEE Trans. Aerosp. Electron. Syst., vol. 52, no. 5, pp. 2596-2603, Oct. 2016.

[13] Q. Chen, B. Tan, K. Chetty, and K. Woodbridge, "Activity recognition based on micro-Doppler signature with in-home Wi-Fi," in Proc. IEEE 18th Int. Conf. e-Health Netw., Appl. Serv., 2016, pp. 1-6.

[14] M.-C. Tang, F.-K. Wang, and T.-S. Horng, "Human gesture sensor using ambient wireless signals based on passive radar technology," in Proc. IEEE MTT-S Int. Microw. Symp., 2015, pp. 1-4.

[15] S. A. Hassani, A. Guevara, K. Parashar, A. Bourdoux, B. van Liempd, and S. Pollin, "An in-band full-duplex transceiver for simultaneous communication and environmental sensing," in Proc. 52nd Asilomar Conf. Signals, Systems, Comput., 2018, pp. 1389-1394.

[16] S. A. Hassani, K. Parashar, A. Bourdoux, B. van Liempd, and S. Pollin, "Doppler radar with in-band full duplex radios," in Proc. IEEE INFOCOMIEEE Conf. Comput. Commun., 2019, pp. 1945-1953.

[17] A. Sabharwal, P. Schniter, D. Guo, D. W. Bliss, S. Rangarajan, and R. Wichman, "In-band full-duplex wireless: Challenges and opportunities," IEEE J. Sel. Areas Commun., vol. 32, no. 9, pp. 1637-1652, Sep. 2014.

[18] B. van Liempd et al., "A+ 70-DBM IIP3 electrical-balance duplexer for highly integrated tunable front-ends," IEEE Trans. Microw. Theory Techn., vol. 64, no. 12, pp. 4274-4286, Dec. 2016.

[19] T. Vermeulen, B. van Liempd, B. Hershberg, and S. Pollin, "Real-time RF self-interference cancellation for in-band full duplex," in Proc. IEEE Int. Symp. Dyn. Spectrum Access Netw., 2015, pp. 275-276.

[20] D. Korpi, Y.-S. Choi, T. Huusari, L. Anttila, S. Talwar, and M. Valkama, "Adaptive nonlinear digital self-interference cancellation for mobile inband full-duplex radio: Algorithms and RF measurements," in Proc. IEEE Global Commun. Conf., 2015, pp. 1-7.

[21] D. Korpi et al., "Full-duplex mobile device: Pushing the limits," IEEE Commun. Mag., vol. 54, no. 9, pp. 80-87, Sep. 2016.

[22] S. H. Abdelhalem, P. S. Gudem, and L. E. Larson, "Hybrid transformerbased tunable differential duplexer in a 90-nm CMOS process," IEEE Trans. Microw. Theory Techn., vol. 61, no. 3, pp. 1316-1326, Mar. 2013.

[23] A. Goldsmith, Wireless Communications. Cambridge, U.K.: Cambridge Univ. Press, 2005.

[24] C. M. Grinstead and J. L. Snell, Introduction to Probability. Providence, RI, USA: Amer. Math. Soc., 2012.

[25] Xilinx, "MicroBlaze soft processor core," 2020. [Online]. Available: http: //www.xilinx.com/tools/microblaze.html, Accessed on: May 20, 2020.

[26] A. C. Carusone and D. A. Johns, "Analog filter adaptation using a dithered linear search algorithm," in Proc. IEEE Int. Symp. Circuits Syst., 2002, vol. 4, pp. IV-IV.

[27] M. Scarpiniti, D. Comminiello, R. Parisi, and A. Uncini, "Hammerstein uniform cubic spline adaptive filters: Learning and convergence properties," Signal Process., vol. 100, pp. 112-123, 2014.

[28] P. Pascual Campo, D. Korpi, L. Anttila, and M. Valkama, "Nonlinear digital cancellation in full-duplex devices using spline-based Hammerstein model," in Proc. IEEE Global Commun. Conf., Dec. 2018, pp. 1-7.

[29] W. Bernard and D. S. Samuel, Adaptive Signal Processing. Englewood Cliffs, NJ, USA: Prentice-Hall, 1985.

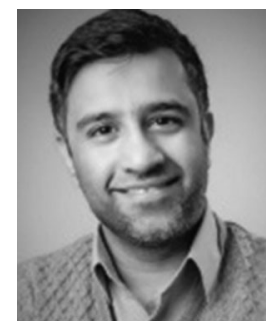

Seyed Ali Hassani (Student Member, IEEE) received the B.Sc. degree in electrical engineering from Arak Azad University, Arak, Iran, in 2008, and the M.Sc. (with distinction) degree in information technology and signal processing from Tampere University of Technology, Tampere, Finland, in 2016.

He then worked as an R\&D Engineer in the industry to gain his professional experience. He is currently a Ph.D. Researcher with KU Leuven, Leuven, Belgium, focusing on context-aware vehicular networking. His main research interests include low-latency networks, radar signal processing, and wireless in-band full-duplex communication. 


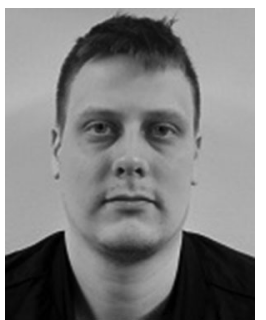

Vesa Lampu received the B.Sc. and M.Sc. degrees in electrical engineering in 2017 and 2019, respectively from Tampere University (formerly TUT), Tampere, Finland, where he is currently working toward the D.Sc. degree.

He has been with the Department of Electrical Engineering, Tampere University since 2018. His research focuses on energy-efficient multiple-inputmultiple-output systems. He has also been involved in research on nonlinear system identification, with applications in full-duplex and digital predistortion

techniques.

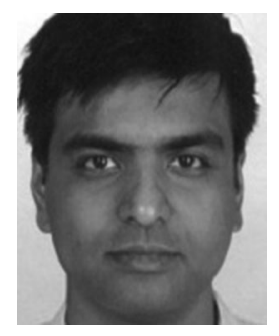

Karthick Parashar received the M.S. degree from the Indian Institute of Technology Madras, Chennai, India, in 2008, and the Ph.D. degree from Inria Rennes-Bretagne Atlantique, Rennes, France, in 2012.

From 2012 to 2014, he was a Postdoctoral Researcher with the Imperial College, London, London, U.K. From 2014 to 2018, he was with the IoT.BE group at IMEC, Leuven, Belgium. He is currently freelancing as an independent researcher/consultant for signal processing algorithms to perform multimodal sensor fusion with cameras (three-dimensional point cloud), radars, and other perception devices. His current research interests include machine learning techniques for multimodal perception, radar signal processing, anomaly detection, analysis of numerical errors, and wireless system design and software architectures.

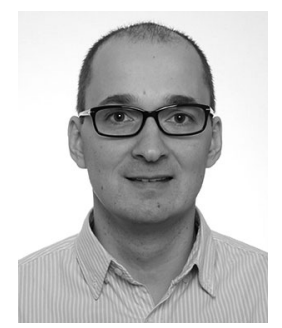

Lauri Anttila (Member, IEEE) received the D.Sc. degree (with honors) in electrical engineering from Tampere University of Technology (TUT), Tampere, Finland, in 2011.

Since 2016, he has been a University Researcher with the Department of Electrical Engineering, Tampere University (formerly TUT). During 2016-2017, he was a Visiting Research Fellow with the Department of Electronics and Nanoengineering, Aalto University, Aalto, Finland. He has coauthored more than 100 refereed articles, as well as three book chapters. His research interests include radio communications and signal processing, with a focus on the radio implementation challenges in systems such as $5 \mathrm{G}$, full-duplex radio, and large-scale antenna systems.

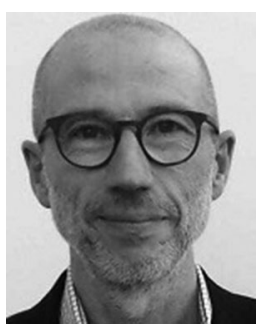

André Bourdoux (Member, IEEE) received the M.Sc. degree in electrical engineering from the Universit Catholique de Louvain-la-Neuve, OttigniesLouvain-la-Neuve, Belgium, in 1982.

He joined IMEC, Leuven, Belgium, in 1998 and is currently a Principal Member of Technical Staff in the Internet of Things research group of IMEC. $\mathrm{He}$ is a System Level and Signal Processing Expert for both the mm-wave wireless communications and radar teams. He has more than 15 years of research experience in radar systems and 15 years of research experience in broadband wireless communications. He holds several patents in these fields. He is the author and co-author of more than 160 publications in books and peer-reviewed journals and conferences. His research interests include the field of advanced signal processing and machine learning for wireless physical layer and high-resolution 3-D/4-D radars.

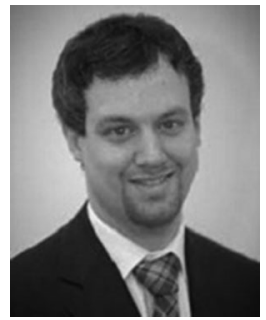

Barend van Liempd (Member, IEEE) received the B.Sc. and M.Sc. degrees in electrical engineering from the Eindhoven University of Technology, Eindhoven, The Netherlands, in 2009 and 2011, respectively, and the $\mathrm{Ph} . \mathrm{D}$. degree from Vrije Universiteit Brussel, Brussels, Belgium, in collaboration with IMEC, Heverlee, Belgium, in 2017.

In 2011, he joined IMEC, working as an R\&D Engineer on multistandard transceivers, until 2014, when he became a Ph.D. Researcher and later a Senior Researcher in 2017. Currently, he leads IMEC's radar IC R\&D activities. He has authored or coauthored more than 30 papers, patents, and patent applications.

Dr. Liempd was the recipient of the 2015 NXP Prize at the European Microwave IC conference.

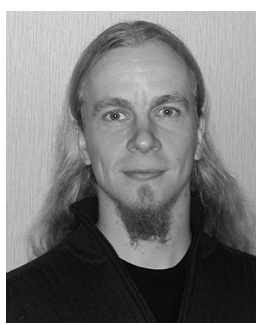

Mikko Valkama (Senior Member, IEEE) received the M.Sc. (Tech.) and D.Sc. (Tech.) degrees (both with honors) in electrical engineering from Tampere University of Technology (TUT), Tampere, Finland, in 2000 and 2001, respectively.

In 2003, he was working as a Visiting Postdoc Research Fellow with the Communications Systems and Signal Processing Institute, San Diego State University, San Diego, CA, USA. He is currently a Full Professor and Department Head of Electrical Engineering with Tampere University (TAU), Tampere, Finland. His research interests include radio communications, radio localization, and radio-based sensing, with particular emphasis on $5 \mathrm{G}$ and beyond mobile networks.

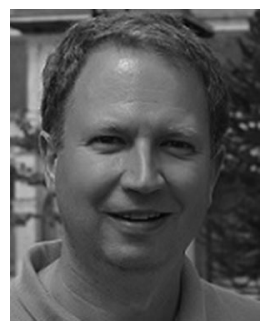

François Horlin (Member, IEEE) received the Electrical Engineering and the Ph.D. degrees from the Universite Catholique de Louvain, Louvain-laNeuve, Belgium, in 1998 and 2002, respectively.

During his studies, he specialized in the field of digital signal processing for communications. In 2002, he joined the Interuniversity Micro-Electronics Center, Leuven, Belgium, where he led the project aiming at developing a fourth-generation wireless communication system in collaboration with Samsung South Korea. In 2007, he became a Professor with the Universit Libre de Bruxelles, Brussels, Belgium. He is an author of a book and a book chapter, a co-author of two patents, and an author or co-author of more than 200 publications in well-recognized journals and conferences.

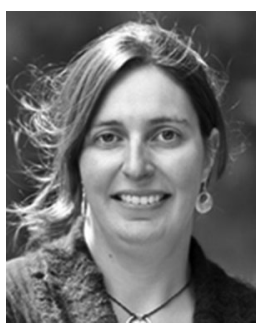

Sofie Pollin (Senior Member, IEEE) received the $\mathrm{Ph} . \mathrm{D}$. degree from KU Leuven, Leuven, Belgium, in 2006.

She continued her research on wireless communication with the University of California, Berkeley, Berkeley, CA, USA. In November 2008, she returned to IMEC to become a Principal Scientist in the green radio team. Since 2012, she has been a Tenure Track Assistant Professor with the Electrical Engineering Department, KU Leuven. Her research centers around networked systems that require networks which are ever more dense, heterogeneous, battery powered, and spectrum constrained. 